\title{
Heparin-induced thrombocytopenia in extra-corporeal membrane oxygenation: epidemiology, outcomes, and diagnostic challenges
}

\author{
Akram M. Zaaqoq ${ }^{1} \cdot$ Robert C. Brammer $^{1} \cdot$ Chee M. Chan $^{1} \cdot$ Andrew F. Shorr ${ }^{1,2}($ D \\ Accepted: 24 July 2021 / Published online: 2 August 2021 \\ (C) The Author(s), under exclusive licence to Springer Science+Business Media, LLC, part of Springer Nature 2021
}

\begin{abstract}
To describe the prevalence of heparin-induced thrombocytopenia (HIT) in patients on extracorporeal membrane oxygenation (ECMO) and to explore ways to improve the diagnostic accuracy of the HIT enzyme-linked immunosorbent assay (ELISA). Retrospective review of all patients needing ECMO between September 2011 and September 2020 who underwent evaluation for HIT while on ECMO. The diagnosis of HIT required a confirmatory serotonin release assay (SRA). Various break points for the optical density (OD) that defines a positive HIT ELISA were examined to estimate their utility as screening tests for HIT. Patient outcomes served as a secondary endpoint. Among 417 ECMO patients, 162 (38.8\%) had a HIT ELISA. Of these, $114(70.4 \%)$ had a subsequent SRA. Although the HIT ELISA was positive at an OD $\geq 0.4$ in $1 / 3^{\text {rd }}$ of subjects, only 15 subjects met criteria for HIT by SRA. Hence, the prevalence of HIT equaled 3.6\%. At an OD $\geq 0.4$ the ELISA had both poor specificity (71.7\%) and accuracy (74.6\%). Changing the definition of the ELISA to an OD $\geq 1.2$ improved both specificity and accuracy with only a limited impact on sensitivity. Nearly $60 \%$ of those with HIT developing during ECMO died. HIT is infrequent in persons requiring ECMO. However, HIT remains associated with substantial mortality. The HIT ELISA as currently implemented performs poorly as a screening test and likely results in the unnecessary overuse of alternatives to unfractionated heparin. Altering the definition of a positive OD improves the HIT ELISA's accuracy.
\end{abstract}

Keywords Diagnosis $\cdot$ Extracorporeal membrane oxygenation $\cdot$ Heparin-induced thrombocytopenia $\cdot$ Mortality

\section{Introduction}

Extracorporeal membrane oxygenation (ECMO) represents a crucial life saving intervention for patients with refractory respiratory failure and/or shock. Since its development several decades ago, ECMO has emerged as an important intervention for a range of patients and can be provided via a veno-venous (VV) or veno-arterial (VA) circuit. The expanded use of ECMO during the current Coronavirus-19 pandemic underscores the developing role for and important value of ECMO [1]. Application of ECMO, however, exposes the patient to a number of potential complications, ranging from hemorrhage to infection. As such, clinicians caring for those undergoing ECMO must remain vigilant in

Andrew F. Shorr

andrew.shorr@gmail.com

1 Pulmonary and Critical Care Medicine Service, Medstar Washington Hospital Center, Washington, DC, USA

2 Medstar Washington Hospital Center, Room 2A-68D, 110 Irving St., NW, Washington, DC 20010, USA their monitoring of these patients. Severe thrombocytopenia exemplifies one common complication of ECMO and occurs in up to $50 \%$ of subjects needing this intervention $[2,3]$. The list of possible causes of thrombocytopenia in ECMO is extensive. Because of the need for systemic anticoagulation with unfractionated heparin (UFH), those treated with ECMO may be at risk for heparin-inducted thrombocytopenia (HIT). HIT results from immunologic-mediated platelet destruction that develops due to exposure to heparin. Despite the ensuing thrombocytopenia, HIT can result in catastrophic venous and/or arterial thromboses [2-4]. The prevalence of HIT in ECMO ranges from 0.5 to $5.0 \%$, depending on the population evaluated $[4,5]$.

HIT represents a unique diagnostic challenge in ECMO. Often utilized clinical scores for defining the pre-test probability of HIT, such as the 4Ts score, have limited sensitivity and specificity in the setting of ECMO [6]. Moreover, the commonly relied upon serum enzyme-linked immunoassay (ELISA) for platelet antibodies also lacks sensitivity and specificity when the question of HIT arises in a subject supported on ECMO [7]. For example, many persons 
needing ECMO, and who lack any clinical evidence of HIT, have circulating platelet factor 4 (PF-4) antibodies prior to ECMO initiation $[8,9]$. Thus, the traditional approach to HIT in scenarios other than ECMO—namely the use of a clinical score and a HIT ELISA — can lead to either the over-diagnosis or under-diagnosis of this potentially life-threatening syndrome [7]. Over-diagnosis could lead to excess cost and risk by causing physicians to switch anticoagulation strategies and employ agents such as bilvaruidin when unnecessary. Conversely, under-diagnosis might result in continued administration of UFH when HIT exists and cause potentially catastrophic outcomes.

One potential alternative approach to this diagnostic challenge relies upon changing the threshold that defines a "positive" HIT ELISA. Traditionally, the HIT ELISA is interpreted based on the optical density (OD) noted by the test. In situations other than ECMO, some have proposed changing the OD cutoff that classifies the assay as positive $[10,11]$. We hypothesized that increasing the OD breakpoint describing a positive result would improve the sensitivity and specificity of the HIT ELISA in patients on ECMO who are suspected of suffering from the HIT syndrome.

\section{Materials and methods}

\section{Overview}

We conducted a retrospective review of subjects undergoing ECMO in whom the treating clinician suspected HIT. Our objectives were to evaluate the screening characteristics of the HIT ELISA for diagnosing HIT in subjects undergoing ECMO and to evaluate alternative thresholds to define an abnormal HIT ELISA. We also aimed to describe the epidemiology of HIT in ECMO. The Medstar Washington Hospital Center Institutional Review Board approved this study. There was no need for informed consent given the retrospective design.

\section{Subjects}

We included all patients undergoing ECMO between September 2011 and September 2020 in whom a HIT ELISA (Genetics Testing Institute Inc. Waukesha, WI) was ordered while on ECMO. We included subjects both on VV and VA ECMO. We required all subjects to have a confirmatory serotonin release assay (SRA, Mayo Clinic Labs, Rochester, MN). Persons in whom a HIT ELISA was ordered but for which there was no subsequent SRA were excluded.

\section{Endpoints}

At our institution, the HIT ELISA is considered positive at an OD of 0.4 based on the manufacturer's recommendation. Specifically, we examined the frequency of positive SRAs at ODs of: $\geq 0.4,0.8,1.0$, and 1.2. The screening attributes of various OD thresholds (eg, sensitivity, specificity, positive predictive value, negative predictive value) represented our secondary endpoint. We also examined rates of in-hospital mortality for patients in whom a HIT ELISA was sent. A positive SRA was presumed to define HIT. A blinded investigator (CMC) reviewed "indeterminate" SRA findings $(\mathrm{n}=4)$ and re-categorized these as either positive or negative after an examination of the medical record. For example, if another cause of thrombocytopenia was identified (eg, major bleeding), the SRA was coded as negative.

\section{Co-variates}

We recorded multiple co-variates so as to explore their relationship with the diagnosis of SRA-positive HIT. Specifically, we noted patient characteristics, ECMO-related variables, and factors associated with the onset of thrombocytopenia. Patient variables of interest included: age, race, and comorbid illnesses (eg, congestive heart failure, hypertension, diabetes, mellitus). ECMO-related measures comprised both the type of ECMO (VV vs VA) employed and the indication for ECMO. We further recorded the timing of the onset of thrombocytopenia, the extent of the platelet nadir, and whether the subject developed a thrombotic complication (eg, deep vein thrombosis, pulmonary embolism, arterial thrombosis).

\section{Statistics}

We compared continuous, parametrically distributed variables with the Student's $t$ test. For categorical variables we utilized Fisher's exact test. For non-parametric variables we relied upon the Wilcoxon rank sum test for comparisons. All tests were unpaired and two-tailed, and a $p$ value of $<0.05$ was presumed to represent statistical significance. We further constructed $2 \times 2$ tables to compute the sensitivity, specificity, positive predictive, and negative predictive values of various OD thresholds as screening tests for a positive SRA. Finally, we computed the area under the receiver operating curve (AUROC) of differing screening thresholds. All analyses were conducted with SPSS 24.0 (IBM, Armonk NY). 


\section{Results}

During the study period, 417 subjects underwent ECMO and $162(38.8 \%)$ had a HIT ELISA sent while on ECMO. Of the 162 patients for whom there was a HIT ELISA, $114(70.4 \%)$ had a subsequent SRA. The 114 patients with confirmatory SRAs did not differ from the 48 individuals in whom there was no follow-on SRA.

The 114 patients with a paired HIT ELISA and SRA represented our final study cohort. The mean age of the study population measured $56.4 \pm 15.6$ years and $64.0 \%$ were male. Approximately $86.0 \%$ of the population underwent VA ECMO. The HIT ELISA was positive at an OD of 0.4 in approximately $1 / 3^{\text {rd }}$ of the study group $(n=42)$. Only 15 members of the final cohort (13.2\%) had a positive confirmatory SRA-suggesting an overall prevalence of HIT in the setting of ECMO of 3.6\%. All but one patient with a positive SRA had a positive HIT ELISA at an OD threshold of 0.4. In the one subject meeting our criteria for the diagnosis of HIT where the ELISA was negative, the
OD value equaled 0.39. Of note, this person's SRA was noted to be strongly positive $(\mathrm{OD}=2.1)$.

Table 1 displays the baseline characteristics of those with and without a positive SRA. Neither age, gender, nor race varied based on the results of SRA testing. Furthermore, the burden of comorbidities was high in the population, with congestive heart failure and hypertension occurring in more than half of all patients. However, there was no difference in the distribution of co-morbid illness as a function of the results of the subsequent SRA.

The indications for ECMO also did not differ between patients diagnosed with HIT and those with a negative SRA (Table 1). The most common reason for needing ECMO in both patients with and without HIT was cardiogenic shock following cardiotomy. We noted no SRA positive results in persons treated with VV ECMO. This difference in the prevalence of HIT between VV and VA ECMO, though, only approached statistical significance $(\mathrm{p}=0.124)$. Table 1 also indicates that neither baseline platelet counts, the platelet count on the day of HIT ELISA testing, nor the nadir platelet value varied between SRA positive and negative subjects.

Table 1 Baseline characteristics

\begin{tabular}{|c|c|c|c|c|}
\hline Variable & $\begin{array}{l}\text { All } \\
(n=114)\end{array}$ & $\begin{array}{l}\text { SRA negative } \\
(\mathrm{n}=99)\end{array}$ & $\begin{array}{l}\text { SRA positive } \\
(\mathrm{n}=15)\end{array}$ & $\begin{array}{l}\mathrm{p} \text { (negative } \\
\text { vs positive) }\end{array}$ \\
\hline Age, years $($ mean $+S D)$ & $56.4 \pm 15.6$ & $56.8 \pm 15.3$ & $53.3 \pm 18.0$ & 0.414 \\
\hline Male, $\%$ & $64.0 \%$ & $63.6 \%$ & $66.7 \%$ & 0.999 \\
\hline Race & & & & 0.348 \\
\hline Caucasian, $\%$ & $36.8 \%$ & $35.3 \%$ & $46.7 \%$ & \\
\hline Black, $\%$ & $37.8 \%$ & $37.3 \%$ & $40.0 \%$ & \\
\hline Other, $\%$ & $25.4 \%$ & $27.3 \%$ & $13.3 \%$ & \\
\hline \multicolumn{5}{|l|}{ Co-morbidities } \\
\hline HTN, \% & $68.4 \%$ & $70.7 \%$ & $53.3 \%$ & 0.233 \\
\hline $\mathrm{CAD}, \%$ & $59.6 \%$ & $61.6 \%$ & $46.7 \%$ & 0.397 \\
\hline $\mathrm{CHF}, \%$ & $64.0 \%$ & $66.7 \%$ & $46.7 \%$ & 0.155 \\
\hline $\mathrm{DM}, \%$ & $48.2 \%$ & $48.5 \%$ & $48.2 \%$ & 0.999 \\
\hline CVA, $\%$ & $15.8 \%$ & $18.2 \%$ & $0 \%$ & 0.123 \\
\hline ECMO type & & & & 0.124 \\
\hline $\mathrm{VV}, \%$ & $14.0 \%$ & $16.2 \%$ & $0 \%$ & \\
\hline VA, $\%$ & $86.0 \%$ & $83.9 \%$ & $100 \%$ & \\
\hline Indications for ECMO & & & & 0.882 \\
\hline$\%$, Post-MI & $21.9 \%$ & $19.2 \%$ & $40.0 \%$ & \\
\hline$\%$, Post-Cardiotomy & $47.4 \%$ & $46.5 \%$ & $53.3 \%$ & \\
\hline$\%$, Cardiogenic Shock & $14.0 \%$ & $16.2 \%$ & 0 & \\
\hline$\%$, Acute Respiratory Failure & $13.2 \%$ & $15.1 \%$ & 0 & \\
\hline$\%$, Pulmonary Embolism & $3.5 \%$ & $3.0 \%$ & $6.7 \%$ & \\
\hline \multicolumn{5}{|l|}{ Platelet Measures } \\
\hline Baseline, $/ \mathrm{ml}$, mean $\pm \mathrm{SD}$ & $211.0 \pm 68.0$ & $214.4 \pm 64.5$ & $223.5 \pm 69.1$ & 0.319 \\
\hline Day of testing, $/ \mathrm{ml}$, mean \pm SD & $53.7 \pm 25.7$ & $56.3 \pm 51.0$ & $50.4 \pm 31.3$ & 0.663 \\
\hline$\% \geq 50 \%$ platelet drop by testing & $93.8 \%$ & $94.8 \%$ & $86.7 \%$ & 0.507 \\
\hline
\end{tabular}

$C A D$ coronary artery disease, $C H F$ congestive heart failure, $C V A$ cerebrovascular accident, $D M$ diabetes mellitus, ECMO extracorporeal membrane oxygenation, $H T N$ hypertension, $M I$ myocardial infarction, $S D$ standard deviation, $S R A$ serotonin release assay, $V A$ venoarterial, $V V$ venonveneous 
Table 2 Testing and outcomes

\begin{tabular}{lllll}
\hline Variable & $\begin{array}{l}\text { All } \\
(\mathrm{n}=114)\end{array}$ & $\begin{array}{l}\text { SRA Negative } \\
(\mathrm{n}=99)\end{array}$ & $\begin{array}{l}\text { SRA Positive } \\
(\mathrm{n}=15)\end{array}$ & $\begin{array}{l}\text { p (Negative } \\
\text { vs Positive) }\end{array}$ \\
\hline HIT ELISA & & & & \\
$\quad$ Positive, $\%$ & $36.8 \%$ & $28.3 \%$ & $93.3 \%$ & 0.001 \\
HIT OD, (mean + SD) & $0.63 \pm 0.82$ & $0.41 \pm 0.52$ & $2.10 \pm 0.90$ & 0.001 \\
$\begin{array}{l}\text { Outcomes } \\
\%, \text { VTE }\end{array}$ & $12.3 \%$ & $11.0 \%$ & & \\
$\%$, Mortality & $58.8 \%$ & $59.6 \%$ & $53.3 \%$ & 0.393 \\
\hline
\end{tabular}

ELISA enzyme linked immunoassay, HIT heparin induced thrombocytopenia, $O D$ optical density, SRA serotonin release assay, VTE venous thromboembolism

Fig. 1 Distribution of optical density as a function of HIT ELISA

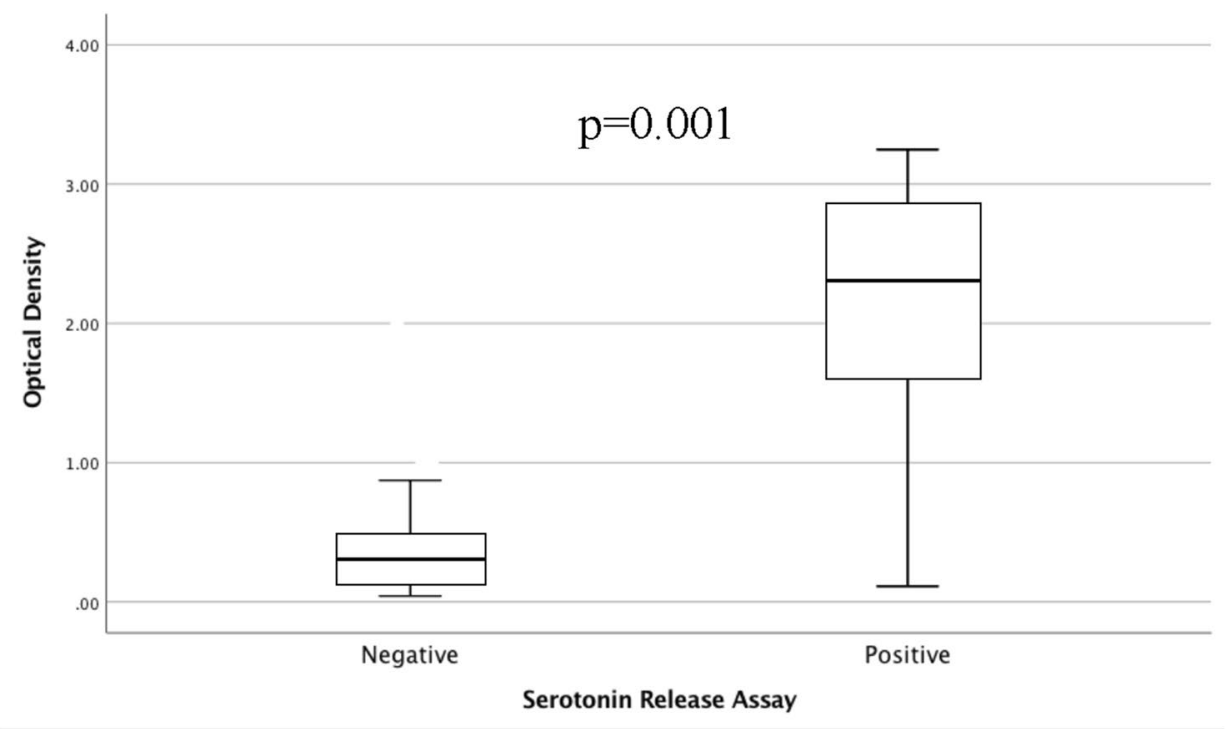

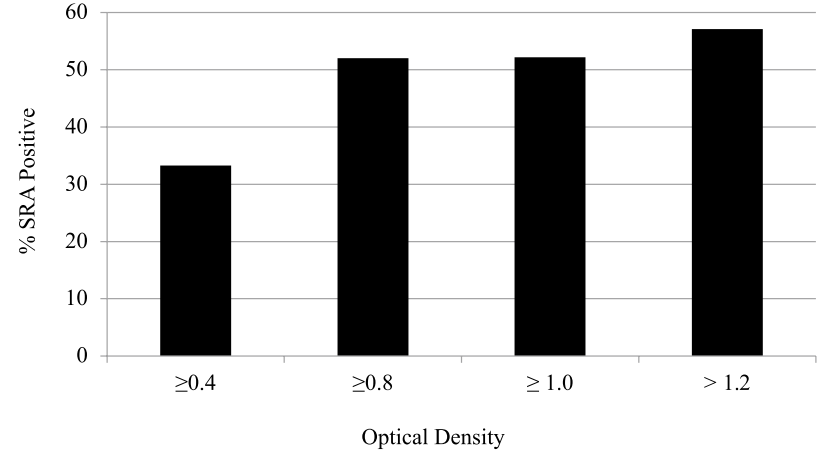

Fig. 2 Relationship between optical density and results of the serotonin-release assay

Not surprisingly, (Table 2; Fig. 1) the mean OD value in those lacking a final diagnosis of HIT measured $0.41 \pm 0.52$ compared to an OD of $2.10 \pm 0.90$ among persons with SRA confirmed HIT $(p=0.001)$. The prevalence of a positive SRA increased as the OD increased (Fig. 2). For example, among patients with an OD of $0.4,33.3 \%$ eventually had a positive SRA compared to $57.1 \%$ of those with an OD of $>1.2(\mathrm{p}=0.048)$.

In terms of outcomes, more VTEs occurred in patients with SRA confirmed HIT but this difference (20.0\% vs. $11.0 \%$ ) was not statistically different (Table 2). Hospital mortality was high in the entire population (58.8\%) but also failed to differ relative to the results of the SRA and a formal HIT diagnosis.

Table 3 displays the screening characteristics of various OD thresholds for predicting a final positive SRA and a diagnosis of HIT. The sensitivity and accompanying negative predictive value of the traditional OD cut point of 0.4 were 93.3\% and $98.6 \%$, respectively. However, this OD threshold yielded an overall test accuracy of only $74.6 \%$, indicating that approximately 1 in 4 subjects would be misclassified with respect to the final diagnosis of HIT. Increasing the cutoff OD to $>1.2$ improved specificity while mildly impairing sensitivity. However, the assay's accuracy as a screening test for a positive SRA improved to nearly $90 \%$. To better illustrate the tradeoffs, we calculate that at the 0.4 level, the HIT ELISA would miss one case of HIT but lead to 28 patients 
Table 3 Screening characteristics

\begin{tabular}{llllll}
\hline OD & Sensitivity (\%) & Specificity (\%) & $\begin{array}{l}\text { Negative predic- } \\
\text { tive value }(\%)\end{array}$ & $\begin{array}{l}\text { Positive predic- } \\
\text { tive value (\%) }\end{array}$ & Accuracy (\%) \\
\hline$\geq 0.4$ & 93.3 & 71.7 & 98.6 & 33.3 & 74.6 \\
$\geq 0.8$ & 86.7 & 87.8 & 97.8 & 52.0 & 87.8 \\
$\geq 1.0$ & 80.0 & 88.9 & 96.7 & 52.2 & 87.8 \\
$\geq 1.2$ & 80.0 & 90.9 & 96.8 & 57.1 & 89.5 \\
\hline
\end{tabular}

$O D$ optical density being over-treated for HIT. Conversely, defining the ELISA as positive only at a value of 1.2 or more would result in 2 additional cases of HIT being missed-but the number of over-diagnosed subjects would fall by $67.9 \%$. This translates into an AUROC for an OD of $>1.2$ of 0.855 .

\section{Discussion}

This retrospective review documents that HIT occurs infrequently in patients supported on ECMO. Moreover, the mortality rate for those with SRA confirmed HIT is high. Yet, this rate of death is similar in all ECMO patients who develop severe thrombocytopenia regardless of the presence of HIT. Importantly, the HIT ELISA preforms poorly as a screening test for HIT and has a limited sensitivity and specificity.

Our findings regarding the general prevalence and outcomes associated with HIT confirm the findings of others $[5,9,12]$. Although the frequency of HIT in prior reports has varied based on a multitude of factors ranging from the population screened to the assays used to confirm the diagnosis, a recent meta-analysis indicates that HIT arises in $3.7 \%$ of those on ECMO [2]. Specifically, Jiritano and co-workers reviewed 21 studies and reported that the rate of HIT varied from less than $1 \%$ to as high as $22 \%$ [2]. In pooling these findings, they concluded that approximately 1 in 27 ECMO subjects is diagnosed with HIT [2]. This rate is strikingly close to the prevalence we observed of $3.6 \%$. It is important to note, however, that our estimated prevalence of HIT reflects a lower bound and is likely conservative. Some patients for whom a clinician ordered a HIT ELSIA but for whom there is no confirmatory SRA may have had HIT. Additionally, we may have missed some instances of HIT in those never evaluated for HIT. Furthermore, the crude, all-cause mortality rate we report aligns with the observations of others and thus indicates the generalizability of the population we studied.

We noted no cases of HIT among patients on VV ECMO. Although the difference in the prevalence of HIT between VV and VA ECMO only approached statistical significance, this observation, nonetheless, merits comment. First, our data should not lead to the conclusion that HIT cannot occur in VV ECMO. Rather, the rate of HIT in VV ECMO may be lower than that seen in VA ECMO. Second, other investigators have described differences in the presentations of and outcomes related to HIT as a function of the type of circuit utilized. For example, Choi et al. commented that those on VA ECMO experience more severe thrombocytopenia and more arterial thromboses in the setting of HIT [12]. Pabst and colleagues, in a review of their experience with HIT in the setting of ECMO, report that VA ECMO patients accounted for nearly $80 \%$ of all the HIT seen. This finding underscores the potential differential prevalence of HIT as a function of the type of support needed [13] . Why might VA ECMO patients face an increased risk for HIT? It may not be directly related to the arterial cannulation and the potential inflammatory and vascular injury ensuing from this. More likely, the need for VA ECMO serves as a surrogate marker that identifies a cohort of subjects who have already experienced extensive exposure to UFH. That the main indication for VA ECMO in the present study was the need for cardiopulmonary support following cardiotomy substantiates this supposition. In essence, it is not VA ECMO per se that is the concern. Rather, it may be that all the factors attendant to the care of the post-cardiotomy patient in refractory shock are what increase the risk for HIT. Larger studies will be needed to disentangle the relationship between VA ECMO, post-cardiotomy ECMO, and the incidence of HIT. More granularity on this topic will facilitate improved diagnostic and risk-stratification strategies.

Our results additionally document the limitations of the HIT ELISA and how this assay potentially complicates the care of those on ECMO suspected of HIT. Although the HIT ELISA has a good negative predictive value, its poor positive predictive value indicates that reliance on the HIT ELISA in the setting of ECMO certainly leads to a potential over-diagnosis of this syndrome. Overdiagnosis of HIT exposes the subject to the risks of anticoagulation with more difficult to utilize agents while one awaits a confirmatory SRA. For instance, reliance on molecules such as argatroban and bilvalrubin possibly confers a higher risk for bleedingwhich can be catastrophic in ECMO. Furthermore, non-UFH anticoagulants are substantially more costly. It is important to note that the threshold OD that defines a positive HIT ELISA derives from studies in normal blood donors yet 
potentially $40 \%$ of persons needing ECMO produce antiplatelet antibodies before even being placed on an ECMO circuit [7]. The combination of these facts underscores why it appears prudent to re-evaluate the role of the HIT ELISA. Some have suggested increasing the OD breakpoint that demarcates a positive result. For example, Chan et al. prospectively evaluated 496 critically patients evaluated for HIT [10]. They noted that increasing the OD threshold to 1.0 from 0.4 maintained the test's sensitivity but increased both the specificity and positive predictive value significantly. In cardiac surgery, other researchers have reached similar conclusions [11]. In a review of 99 patients with suspected HIT following cardiac surgery, changing the definition of a positive HIT ELISA to an OD of $\geq 1.0$ also enhanced specificity without compromising sensitivity [11]. That we observed an improvement in overall diagnostic accuracy from $75 \%$ to approximately $90 \%$ underscores the logic for adjusting the OD measure delineating an abnormal result. More specifically, an OD of 1.2 reduced the number of overdiagnoses and yielded an AUROC of 0.855 , suggesting good screening test operating characteristics. However, adoption of an elevated OD does not preclude the need for a confirmatory SRA. In our cohort, the higher OD breakpoint resulted in a positive predictive value below $60 \%$. Alternatively, the ability to obtain SRA results more quickly, as is the case in some countries, would preclude the need for the HIT ELISA and its attendant limitations entirely. Before implementation of any new definitional criteria for the HIT ELISA, our observations require validation in larger groups of ECMO patients.

The present study suffers from multiple limitations. First, its retrospective design exposes it to various forms of bias. We attempted to address the impact of this by focusing on endpoints not prone to misinterpretation, such as the results of the diagnostic SRA. Likewise, more than $70 \%$ of those with suspected HIT had confirmatory SRA testing, while those who lacked an SRA did not clinically differ from persons undergoing the added testing. Second, our findings derive from a single center. As such our results may not be generalizable to other settings. Third, our sample size was limited. Thus, the statistical power inherent in our analyses is likely inadequate. Yet, compared to other single center reports, our sample size was larger than most. Finally, we assumed HIT requires the presence of a positive SRA. However, experts have noted selected cases of SRA-negative HIT.

In conclusion, HIT occasionally complicates the use of ECMO. Mortality rates in ECMO- associated HIT are high but are similar to those seen in others on ECMO with significant thrombocytopenia. The standard screening test for HIT, as presently utilized, performs poorly at it designated task. This fact and the noted lack of validity associated with clinical HIT risk stratification tools suggests a new paradigm is needed in ECMO. One potential alternative approach is to re-evaluate the OD measures that define a positive HIT ELISA result. Raising the threshold OD for HIT screening in those on ECMO enhances diagnostic accuracy.

Author contributions AZ designed the analysis, collected the data, and drafted and edited the paper. RB conceived of the study, collected that data, contributed to the analysis, and edited the paper. CC contributed to data analysis, performed the analysis, wrote the paper, and edited the paper. AS conceived of the study, conducted the analysis, wrote the paper, and edited the paper.

Funding This research did not receive any specific grant from funding agencies in the public, commercial, or not-for-profit sectors.

Data availability Data are available from the authors upon request.

\section{Declarations}

Conflict of interest The authors have no disclosures relevant to the topic of this manuscript. The authors have no financial conflicts of interests and no competing interests.

Ethical approval The Medstar Washington Hospital Center Institutional Review Board approved this study.

\section{References}

1. Zhu Y, Zhang M, Zhang R, et al (2021) Extracorporeal membrane oxygenation versus mechanical ventilation alone in adults with severe acute respiratory distress syndrome: a systematic review and meta-analysis. Int J Clin Pract e14046.

2. Jiritano F, Serraino GF, Ten Cate H et al (2020) Platelets and extra-corporeal membrane oxygenation in adult patients: a systematic review and meta-analysis. Intensive Care Med 46:1154-1169

3. Moreno-Duarte I, Ghadimi K (2020) Heparin induced thrombocytopenia for the perioperative and critical care clinician. Curr Anesthesiol Rep 29:1-11

4. Althaus K, Straub A, Häberle T et al (2020) Heparin-induced thrombocytopenia: dignostic challenges in intensive care patients especially with extracorporeal circulation. Thromb Res 188:52-60

5. Hogan M, Berger JS (2020) Heparin-induced thrombocytopenia (HIT): Review of incidence, diagnosis, and management. Vasc Med 25:160-173

6. Sullivan J, Bak E, Sullivan MJ et al (2020) Predictive value of scoring tools in determining heparin-induced thrombocytopenia in patients on extracorporeal membrane oxygenation. Perfusion 35:378-383

7. Warkentin TE (2019) Laboratory diagnosis of heparin-induced thrombocytopenia. Int J Lab Hematol 41(Suppl 1):15-25

8. Vayne C, May MA, Bourguignon T et al (2019) Frequency and clinical impact of platelet factor 4-specific antibodies in patients undergoing extracorporeal membrane oxygenation. Thromb Haemost 119:1138-1146

9. Selleng S, Selleng K (2016) Heparin-induced thrombocytopenia in cardiac surgery and critically ill patients. Thromb Haemost 116:843-851

10. Chan CM, Woods CJ, Warkentin TE et al (2015) The role for optical density in heparin-induced thrombocytopenia: a cohort study. Chest 148:55-61 
11. Chan CM, Corso PJ, Sun X et al (2011) Evaluating the role for the optical density in the diagnosis of heparin-induced thrombocytopenia following cardiac surgery. Thromb Haemost 106:934-938

12. Choi JH, Luc JGY, Weber MP et al (2019) Heparin-induced thrombocytopenia during extracorporeal life support: incidence, management and outcomes. Ann Cardiothorac Surg 8:19-31

13. Pabst D, Boone JB, Soleimani B et al (2019) Heparin-induced thrombocytopenia in patients on extracorporeal membrane oxygenation and the role of a heparin- bonded circuit. Perfusion 34:584-589

Publisher's Note Springer Nature remains neutral with regard to jurisdictional claims in published maps and institutional affiliations. 